\title{
FASH: Detecting Tiredness of Walking People Using Pressure Sensors
}

\author{
Kenji Yonekawa*1, Takuro Yonezawa*2, Jin Nakazawa*1, Hideyuki Tokuda*1,2 \\ ${ }^{* 1}$ Faculty of Environment and Information Studies \\ ${ }^{* 2}$ Graduate School of Media and Governance \\ Keio University, Japan, \{kenz, takuro, jin, hxt $\} @$ ht.sfc.keio.ac.jp
}

\begin{abstract}
The number of elders who encounter falling accidents has been increasing in the past few decades. Falling accidents could cause major injuries, such as having bruise, breaking bone, and in the worst case, losing life. Therefore, preventing elders from falling accidents is important in order to ensure the quality of life. In this paper, we propose Fatigue Alerting SHoes (FASH), a pair of shoes which detects the fatigue of walking people. There are three effects of fatigue on gait, and FASH system acquires all of them to improve the accuracy of fatigue detection. FASH system uses pressure sensors embedded in a pair of shoes to detect the tiredness, and notifies the user of "Tiredness Scale," a scale we defined in order to show the degree of tiredness. Through the evaluation, we affirmed the availability of using pressure sensor to detect accumulation of tiredness. We also confirmed the difference between the physical tiredness and the subjective feeling toward tiredness, which signifies the importance of acknowledging the user of his/her fatigue.
\end{abstract}

\section{INTRODUCTION}

Every year, approximately thirty percent of elderly population encounter falling accidents, resulting in having bruise, breaking bones, and in the worst case, losing life [1] [2] [3]. Compared to that of the youth, elders have higher chances of getting into falling accidents due to many factors, such as, weaken physical strength, the adverse effect of medicine, and the experience of falling accidents which he/she has encountered in the past [4] [5] [6]. Moreover, elders have high chance of falling without protecting their important body parts, such as, head, neck and bones, because of the weaken reflects, which is one of the traits of the elders. Falling from the head could result in death, and falling from other body parts could result in breaking bone at a great chance, since the elder's bones are much weaker than those of the youth. In addition, the hospitalization cost for injury due to falling accident is much more expensive compared to that of the other causes, such as, traffic injury, machine injury, etc. [7]. Falling accident is a major threat for the elders, therefore, it is strongly desired to be prevented.

It is proven that a third of people were walking when they encountered falling accidents [8], thus, preventing them from the accidents can save the most number of people from getting injured or being killed. It is claimed that nearly thirtyfive percent of elders fell for not fulfilling the environmental demands [9], which is caused by either extrinsic or intrinsic cause [3] [10]. The extrinsic cause is an environmental factor which may cause people to fall, and the intrinsic cause is as person's physical characteristics. In order to reduce the effects of extrinsic cause, removing environmental needs, for example, removing steps and rocks from a street, is required. Since it is impossible to remove all kinds of obstacles, for instance, impossibleness to remove all rocks from public place, the effect of intrinsic cause must be got rid of, or reduced.

The intrinsic cause can be divided into two factors: static factor and dynamic factor. Static factor consists of weaken muscle, physical strength, and reflex as a result of getting old [11]. On the other hand, dynamic factor includes losing balance and motility function as a result of getting tired [12] [13] [14]. The influence of aging takes place gradually, which allows elders to be aware of it, meanwhile, the effect of fatigue on gait is difficult to recognize. Moreover, fatigue has a great impact on gait, and it arises rapidly [15]. This indicates the importance of fatigue detection, and thus, we aim to prevent the falling accidents by detecting fatigue of walking people, and alerting the user of it. In addition, youths lacking physical activities are resulting in various health problems, such as obesity and metabolic syndrome, and informing his/her tiredness can be influential.

The effects of fatigue on gait are, 1) lower height of raised legs [12], 2) slower walking tempo [16], and 3) losing balance [13]. This, in turn, shows that detecting these features can be used to acquire fatigue. Several research [17] [18] acquire the balance of the user, which is only one of the effects of fatigue on gait. The use of video cameras [19], can also be used to detect the effect of fatigue on gait. These research either attaches devices to human body, or embeds cameras into the environment, which is not suitable to be used in everyday life. In order to solve this problem, we propose a new fatigue detection method using pressure sensors implanted in a pair of shoes, FASH: Fatigue Alerting SHoes (See Fig. I). FASH system has two features: 1) installation simplicity and 2) accurate detection of fatigue. FASH system needs no device attached to the user's body, because the devices and sensors are embedded in a pair of shoes. FASH system obtains all three effects of fatigue on gait in order to detect tiredness, which is considered to improve the accuracy of fatigue detection.

The rest of this paper is organized as follows. In Section II, we present how other research detect fatigue, and discuss which sensors or devices should be used for fatigue detection by comparing them. In Section III, we introduce and discuss the result of pre experiment which we conducted in order to check the validity of using pressure sensors to detect 


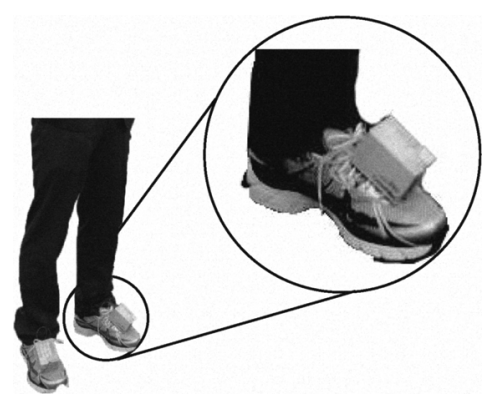

Figure 1. Person Wearing FASH

fatigue. We then explain the implementation of FASH system in Section IV, and evaluate its validity in Section V. The paper concludes with Section VI.

\section{Problem Statement}

This section describes how other research detect fatigue using a specific sensor or device. Then it discusses which sensor or device is suitable for fatigue detection in everyday life by comparing them.

\section{A. Related Work}

Several research have proposed methods to detect fatigue. Balance acquirement is the most common way to detect fatigue [17] [18]. In [17], accelerometer is implanted in the device attached to the user's limbs, which acquires the balance of the user and notify the user of it by vibrating another device attached to his/her head. In [18], accelerometers are hung beside the user's body, and notifies the user of his/her balance through a small handheld device equipped with LCD. These research aim to acquire the balance of the users, but not the legs height nor the walking tempo, which are also the effects of fatigue on gait. Not acquiring all the effects of fatigue on gait is considered to result in less accuracy of fatigue detection. Ubiquitous services are preferred to be implemented in a way that the users' surroundings would not be affected, nor destroyed by sensors or devices. It is also important that the users would not be aware of them. However, the research mentioned above attaches the device to the body, or has the device hung from the body, which is not suitable to be used in everyday life.

Users are made to walk on the specific place, and their gait is being recorded with video cameras to extract the body parts movement [19]. Although this research is not aiming to detect the effect of fatigue, the method of using video cameras to analyze user's gait can also be used to obtain the effects of fatigue on gait. This method most likely be able to obtain user's gait more precisely than the research referred above. However, the cameras have to be embedded in a specific environment, therefore, this method is not appropriate to be used in everyday life.

Research aiming for fatigue detection [20] only distinguishes if the user is tired or not, and it cannot be used to know how tired the user is. It is important for the users to know the degree of tiredness in order to be aware of it's effects on gait as much as possible. In order to solve this problem, notifying the user of his/her magnitude of tiredness is required. It is also important to encourage users to spend time on exercise by acknowledging them of their fatigue.

\section{B. Design Issues}

Mobile devices for healthcare should be easily installed, and should not bother users when in use. Installation simplicity is important when used by elders. Thus, those devices are desired to be embedded in something that user wears, for example, clothes, hats and shoes. Moreover, the data must be as accurate as possible in order to be evaluated correctly, especially for healthcare devices, since it may concern user's life.

Table I illustrates the comparison between accelerometer, camera, and pressure sensor when they are used for fatigue detection. The height of legs, walking tempo, and body balance corresponds to the three influence of fatigue on gait respectively, and usability represents the easiness of installation and the suitableness of it for everyday life. We compared accelerometer attached to different body parts; waist, feet, and both waist and feet. Accelerometer attached to waist, and camera represents research mentioned in Section II-A [17] [18] [19]. Pressure sensor is compared to the other devices, because it is thought to be able to be embedded in shoes without any installation difficulties, and able to acquire the three effects of fatigue on gait.

Height of legs cannot be acquired only by attaching accelerometer to waist, however, walking tempo and body balance can be obtained. Attaching accelerometer to feet enables acquiring the height of legs and walking tempo, but not the balance of the body. If the accelerometer is attached to waist and feet, the three effects of fatigue on gait can be obtained by covering the undetectable feature of each other. As brought up in Section II-A, using camera to record and analyze the body segments movement can obtain three effects of fatigue on gait.

Attaching sensor or device to user's body is not suitable for the use in everyday life, since it needs user's attention whenever, and wherever they go, for instance, user must make sure that the device does not get hit or fall. Implementing sensor to feet, for example, embedding accelerometer or pressure sensor into shoes permits user to live his/her everyday life without any attention to it. However, as shown in Table I, embedding accelerometer into shoes does not fulfill the requirements. Using camera is not appropriate for everyday life; cameras must be embedded in the environment, and cannot be used in

Table I

COMPARING SENSORS FOR DETECTING TIREDNESS

\begin{tabular}{|c|c|c|c|c|}
\hline & height of legs & tempo & body balance & usability \\
\hline $\begin{array}{r}\text { accelerometer } \\
\text { waist } \\
\text { feet }\end{array}$ & $\begin{array}{c}\text { poor } \\
\text { good } \\
\text { good }\end{array}$ & $\begin{array}{c}\text { fair } \\
\text { good } \\
\text { good }\end{array}$ & $\begin{array}{c}\text { good } \\
\text { poor } \\
\text { good }\end{array}$ & $\begin{array}{c}\text { poor } \\
\text { good } \\
\text { poor }\end{array}$ \\
\hline camera & good & good & good & poor \\
\hline pressure sensor & good & good & good & good \\
\hline
\end{tabular}


other places. Pressure sensor is thought to be the only sensor which can acquire all three effects of fatigue on gait without any influence on everyday life, therefore, FASH system uses pressure sensor to detect fatigue. Moreover, the pressure sensor is as thin as 0.018 inches, thus, it is considered that the whole system can be embedded under the sole of a shoe. However, the validity of using pressure sensors to detect fatigue is not proved, hence, we held a pre experiment in order to evaluate it and the result of it is explained in Section III.

\section{PRE EXPERIMENT}

This section conducts an experiment to prove the availability of pressure sensors to obtain the effects of fatigue on gait.

In order to evaluate the pressure data correctly, the value of it is desired to have a large difference between the minimum and the maximum pressure value. We embedded pressure sensors to six places in a pair of shoes: under the heel, arch of the foot, the opposite side of arch of the foot, base of the thumb, base of the middle finger, and base of the pinky finger. In order to check the change in pressure value, a nineteen years old man wore this shoes and walked freely on a flat floor for about five minutes. As a result, we observed the slightest change in pressure value from the sensor embedded under the arch of the feet. On the other hand, the most differences in pressure value were observed from the sensors implanted under the heel, and base of the thumb. Therefore, we embed pressure sensors under the heel and toe to detect fatigue.

We made four male, aged nineteen to twenty-two, run at $10 \mathrm{~km} / \mathrm{h}$ for thirty minutes on a treadmill in order to make them tired. The pressure value is acquired from pressure sensors implanted in a pair of shoes, before and after the run when walking freely on a flat floor.

Fig. 2(a) and 2(b) illustrates the mean pressure value of toe and heel against time, before and after the run respectively. Each wave indicates the pressure value transition of each step. We observed three differences in pressure value before and after fatigued described as follows:

1) decrease in maximum pressure

2) increase in time of foot on the ground

3) transition of pressure in between feet

The dashed line indicates the mean value of maximum pressure of each step, which is approximately $70 \mathrm{kgm} / \mathrm{s}^{2}$ before fatigued, and $65 \mathrm{kgm} / \mathrm{s}^{2}$ after fatigued. This shows

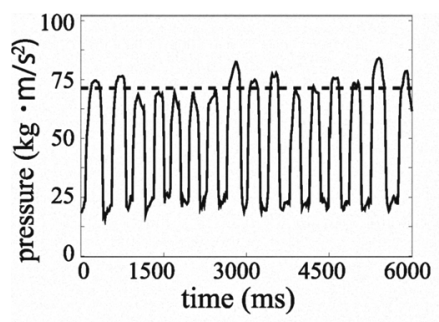

(a) before fatigued

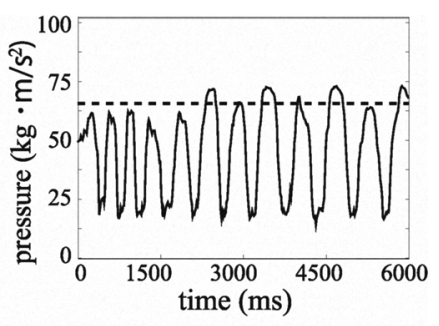

(b) after fatigued
Figure 2. Pressure value vs. time before and after fatigued
Table II

COMPARING RATE OF CHANGE

\begin{tabular}{|c|c|c|c|}
\hline & maximum pressure & $\begin{array}{c}\text { time of feet } \\
\text { on the ground }\end{array}$ & $\begin{array}{c}\text { ratio of pressure value } \\
\text { between toe and heel }\end{array}$ \\
\hline participant A & 0.929 & 1.244 & 0.903 \\
\hline participant B & 0.932 & 1.176 & 0.919 \\
\hline participant C & 0.942 & 1.167 & 0.902 \\
\hline participant D & 0.940 & 1.165 & 0.903 \\
\hline mean & 0.936 & 1.188 & 0.907 \\
\hline
\end{tabular}

that, by getting tired, the maximum pressure value goes down, which signifies that the impact of each step being reduced. It is considered that one of the effects of fatigue on gait, lower height of raised legs results in decrease in maximum pressure of each steps.

The difference in frequency of the wave between before and after fatigued is significant. We observed longer time taken for each steps after fatigued compared to that of before fatigued: average time taken for each step is roughly about $400 \mathrm{~ms}$ and $500 \mathrm{~ms}$ before and after fatigued respectively. Taking longer time for each step means slower walking tempo, which is one of the effects of fatigue on gait.

By comparing pressure value of sensors placed under the heel and toe before and after fatigued, we observed the transition in pressure value in between the feet. It is revealed that the weight of the body tends to shift towards back by getting tired: the pressure value of heel increases, meanwhile, that of toe decreases after being fatigued. Shinkoda states that the more weight applies to the back of the feet, the more unstable the human body gets [21]. This indicates that the weight transition we observed in the experiment, the weight shift toward backside of feet, is equivalent to losing balance.

Table II shows the "rate of change" of each items before and after fatigued. We define "rate of change" as the ratio between the value before and after fatigued for three effects of fatigue on gait. For example, participant A's maximum pressure value after fatigued had become $92.9 \%$ of that of before fatigued. The differences in pressure value is observed in every four participants in the similar way, which verifies the use of pressure sensors to detect fatigue of walking people. The data of participant A's time of feet on the ground, and participant B's ratio of pressure value between toe and heel has larger difference compared to those of the other participant's. This signifies that not using all three effects of fatigue may result in the misevaluation of tiredness. Thus, it is considered that the research mentioned in Section II-A have possibility to misevaluate the data. On the other hand, FASH system uses all three effects of fatigue on gait to detect tiredness, which increases the accuracy of fatigue detection.

\section{FASH SYSTEM}

This section describes the system of FASH. It consists of definition of "Tiredness Scale", and implementation of FASH.

\section{A. Tiredness Scale}

We define "tiredness scale" as a value from 0 to 100 , which represents the magnitude of the user's tiredness. Tiredness 
scale is calculated using the three differences in pressure value explained in Section III. Fatigue scale $\hat{T}$ is calculated using the following Equation (1)(2).

$$
\begin{array}{r}
\hat{T}=100\left(\frac{w_{1}\left(a-a_{t}\right)}{a-x a}+\frac{w_{2}\left(b_{t}-b\right)}{y b-b}+\frac{w_{3}\left|c-c_{t}\right|}{c-z c}\right) \\
w_{1}+w_{2}+w_{3}=1
\end{array}
$$

$a, b$, and $c$ signifies the maximum pressure, time of feet on the ground, and ratio of pressure value between toe and heel before being fatigued respectively. $a_{t}, b_{t}$, and $c_{t}$ each stands for maximum pressure, time of feet on the ground, and ratio of pressure value between toe and heel at a specific period of time. Nardon et al. claims that the body tends to be unstable when being fatigued [13], indicating human body may lean forward as a result of getting tired, thus we used absolute value for $c-c_{t} . w_{1}, w_{2}$, and $w_{3}$ indicates the weight, which sums up to 1. $x, y$, and $z$ each stands for the rate of change of the three effects of fatigue on gait.

The value of rate of change can be determined by two ways, either gathering pressure data of each user to calculate the value before using FASH, or using predefined value. By gathering the pressure data, the rate of change becomes the most suitable rate for any user, however, making the user fatigued in order to obtain the data is not realistic since it requires tremendous time and effort before using FASH. On the other hand, using predefined value for the rate of change allows users to use FASH without any preparation, although, the rate of change may not be suitable for some users. Nishiyama et al. concludes that the age and sex does not affect the amount of muscle used when walking, except for the rectus muscle [22]. This implies that the rate of change of a specific user can most likely be applied to other users. In order to determine the validity of using predefined value of rate of change, we held an experiment explained in Section III.

Table II shows the rate of change of four participants. The value of rate of change turned out to be almost the same, expect for participant A's time of feet on the ground, which is thought to be the outlier. This proves the validity of using predefined value of rate of change to detect fatigue, thus, we use the average of four participant's rate of change in FASH system. The rate of change of maximum pressure, time of feet on the ground, and ratio of pressure value between toe and heel, is set to $0.936,1.188,0.907$, and they are substituted into $x, y$, and $z$ of the Equation (1) respectively. The weight, $w_{1}, w_{2}$, and $w_{3}$, is set to one third until we define the best weight for them. The equation for calculating tiredness scale $T$, after substituting the defined values into the Equation (1) is stated as follows:

$$
T=100\left(\frac{\frac{1}{3}\left(a-a_{t}\right)}{a-0.936 a}+\frac{\frac{1}{3}\left(b_{t}-b\right)}{1.188 b-b}+\frac{\frac{1}{3}\left|c-c_{t}\right|}{c-0.907 c}\right)
$$

\section{B. Implementation}

Fig. 3 illustrates the architecture of FASH system. FASH consists of four pressure sensors, two Imote2s [23] and a NUTS. The pressure sensor used in FASH system can acquire

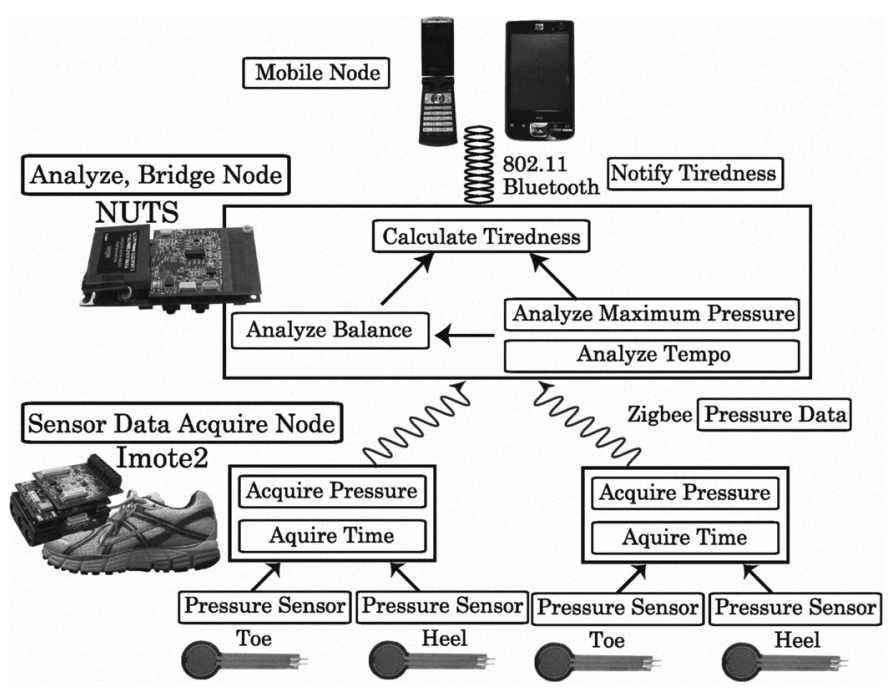

Figure 3. FASH Architecture

pressure values from 0 to $100 \mathrm{kgm} / \mathrm{s}^{2}$. Fig. 4 shows the actual implementation of pressure sensors in a shoe. The white circle indicates the place where the pressure sensors are implanted. Imote 2 is an intel mote, which has a sensor board (3-axis accelerometer, temperature, luminescence, humidity sensor, and analog input,) CPU, battery, and ZigBee module. NUTS is a device developed in our Lab., which has sensor board (3-axis accelerometer, temperature, luminescence, GPS, RFID, etc.,) gumstix [24] as CPU, I/Os (microphone, earphone, LCD,) battery, and wireless module (WiFi, Bluetooth, ZigBee.)

The pressure sensors are inserted under the sole of a pair of shoes. Imote2s are attached to both right and left shoes, and two pressure sensors are connected to each of them. Imote 2 acquires the pressure data from the pressure sensors at 50 samples per second, and sends the data to NUTS over ZigBee. The frequency of pressure data acquisition is tested, and it is concluded that 50 samples/second is the minimum frequency which ensures the obtainment of data for fatigue detection. The higher the frequency becomes, the more power it consumes. Thus, the frequency is set to the minimum in order to extend the time of FASH's life, so that it is suitable for the use in everyday life. We confirmed nearly 10 percent packet loss rate when Imote 2 sends pressure data to NUTS over ZigBee at $50 \mathrm{~Hz}$ due to packet interference between two Imote2s and

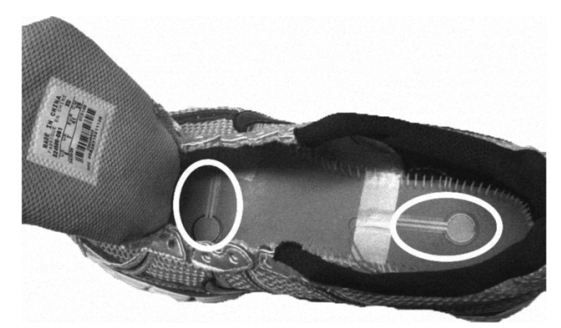

Figure 4. Pressure Sensors in Shoe 
NUTS. Therefore, Imote 2 reduces the sending frequency to approximately $1 \mathrm{~Hz}$ by extracting the required pressure data for fatigue detection, and putting multiple extracted data into one packet. As a result, the packet loss rate becomes about 1 percent, which is permissible rate for the fatigue detection.

NUTS is used for detecting fatigue, calculating tiredness scale, and notifying the tiredness scale to the user. However, NUTS is used as a bridge node, and is not needed if it is only used for detecting fatigue or calculating tiredness scale, because they can be done in Imote2 instead. Note that this FASH system architecture is a prototype: the devices and pressure sensors can be made much smaller if they are optimized for FASH. Each time NUTS receives a packet from Imote2, it evaluates fatigue using three differences in pressure value, and calculates the tiredness scale. Then it notifies the user of it over WiFi, Bluetooth, ZigBee, or through I/Os such as sound and LCD. Although we currently alert the user immediately after the calculation of tiredness scale through e-mail, we are now considering notification using beep, vibration, music, etc.

\section{EVALUATION}

In this section, we evaluate the validity of fatigue detection using predefined value for rate of change, by showing the result of valuation and discussing it.

We applied FASH to three male, twenty to twenty-two years old, and have them follow the subsequent steps using treadmill:

1) walk for 1 minute at $2 \mathrm{~km} / \mathrm{h}$, and obtain the pressure data

2) run for 10 minutes at $10 \mathrm{~km} / \mathrm{h}$

3) walk for 1 minute at $2 \mathrm{~km} / \mathrm{h}$, and obtain the pressure data

4) repeat the steps 2) and 3) once

We used the pressure data acquired in step 1) as the pressure data before fatigued, and compared it against the data acquired in step 3) and step 4). We used this sequence in order to check if the tiredness scale increases as the user gets tired, and if FASH is applicable for a new user, using the predefined value of the rate of change. According to [25], running at $10 \mathrm{~km} / \mathrm{h}$ uses approximately three times as much muscle as walking at $2 \mathrm{~km} / \mathrm{h}$. In order to make the user tired, making him/her walking is the best way, however, this shows the effectiveness of running instead of walking. Running is less time consuming than walking, thus we made participants run, in order to acquire data as much as possible in short time.

Fig. 5 illustrates the graph of tiredness scale vs. number of data. The wavy line indicates 10 minutes run, and the solid line shows the tiredness value calculated in 1 minute walk.

The solid line always has greater value on the right side of a wavy line compared to that on the left side, which means that the value of tiredness scale increases after running. The increase in the value of tiredness scale by getting fatigued signifies the validity of the method used for tiredness detection, and the equation to calculate tiredness scale.

We confirmed the difference in physical fatigue, and subjective feeling toward tiredness. All participants run the same amount of time at same speed twice, and the tiredness scale calculated from the acquired data has raised about the same amount after each run. In spite of that, participant A and C claimed that on the second run, they got half as tired as the first run, and participant B affirmed that he got more tired on the second run compared to the first run. This represents that the users have difficulties to realize their tiredness, thus, it is important to notify them of it to acknowledge the degree of tiredness. Moreover, this indicates the validity of FASH acknowledging the users of their degree of fatigue.

We used predefined values for the rate of change in the tiredness scale calculation as shown in Equation (3), and we confirmed the validity of fatigue detection method. This indicates that the rate of change of a specific user can be applied to other users. However, as of right now, the rate of change is calculated from four participants, thus, the greater number of data should be acquired to define most proper value.

\section{CONCLUSION}

This paper proposes Fatigue Alerting SHoes (FASH), a pair of shoes which detects the tiredness of the walking people and alerts the users of it. Rather than using accelerometer or camera, we use pressure sensors to detect the tiredness, and confirmed the effectiveness of using pressure sensors for fatigue detection.

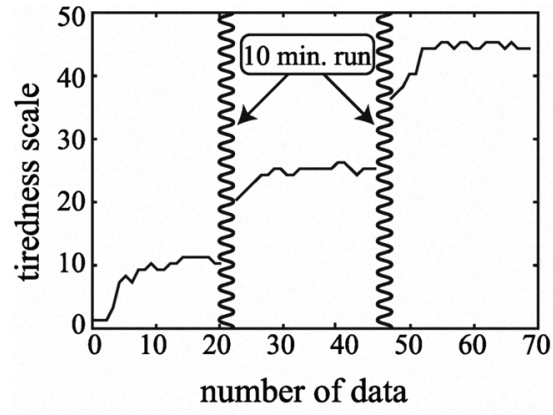

(a) Participant $\mathrm{A}$

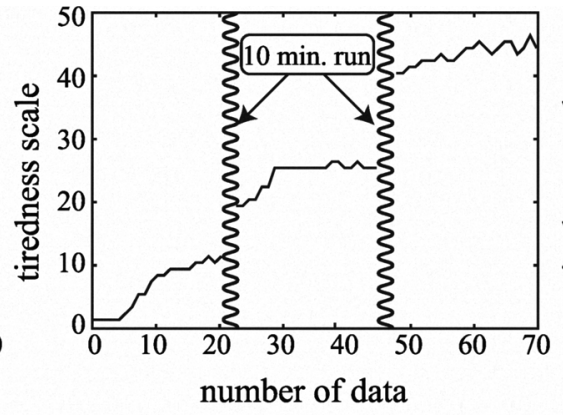

(b) Participant B

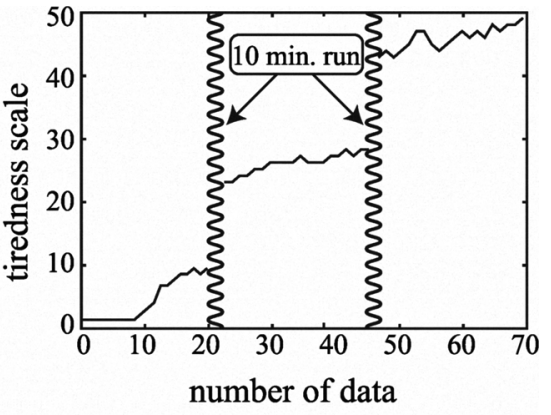

(c) Participant $\mathrm{C}$

Figure 5. Tiredness Scale vs. Number of Data 
There are three effects of fatigue on gait, 1) lower height of raised legs [12], 2) slower walking tempo [16], and 3) losing balance [13], and FASH system obtains all of them to detect fatigue, while research using accelerometer only uses one of them [17] [18]. Moreover, FASH system is suitable for everyday life compared to other research [17] [18] [19], because it embeds pressure sensors in a pair of shoes, which allows users to wear them without any interference. We define "Tiredness Scale", a scale which represents how tired the user is, and propose the equation to calculate it. This scale allows FASH system to inform the user of the magnitude of the effects of fatigue on walking. Through the evaluation, we confirmed the validity of using predefined value for the rate of change, tiredness scale and the equation for its calculation. We also confirmed the importance of informing the user of his/her fatigue, since they have difficulties to recognize their own physical tiredness.

There are a few concerns remain in the current design and implementation of FASH system. In Equation (3), we defined the weight as one third, however, it should be investigated in order to maximize the accuracy of fatigue detection. As of right now, the value of rate of change for the three influence of fatigue on gait are based on the data obtained from four male aged from nineteen to twenty-two. However, the difference in age and sex may cause the value of rate of change to be different. Therefore, more number of data should be obtained from various age and different sex in order to define the most suitable value for the rate of change. Although we currently use tiredness as a scale to diagnose the danger of walking, use of other measurements such as energy expenditure, energy consumption, etc. should be considered. Finally, we are presently notifying the users immediately after the calculation of their tiredness scale through e-mail. Although, using e-mail as notification is not proper for some people, especially for the elders. Therefore, the timing and the way of notification should be investigated carefully in order to prevent the users from falling accidents, and encourage the users to do exercise.

\section{ACKNOWLEDGEMENT}

This research is partially supported by the Ubiquitous Service Platform Research of Ministry of Internal Affairs and Communications, Japan.

\section{REFERENCES}

[1] P. Kannus, M. Palvanen, S. Niemi, J. Parkkari, A. Natri, I. Vuori, and M. Jarvinen, "Increasing Number and Incidence of Fall-induced Severe Head Injuries in Older Adults: Nationwide Statistics in Finland in 19701995 and Prediction for the Future," Am. J. Epidemiol., vol. 149, no. 2, pp. 143-150, 1999.

[2] G. Fuller, "Falls in the elderly," American Academy of Family Physicians, 2000.

[3] W. Graafmans, M. Ooms, H. Hofstee, P. Bezemer, L. Bouter, and P. Lips, "Falls in the Elderly: A Prospective Study of Risk Factors and Risk Profiles," Am. J. Epidemiol., vol. 143, no. 11, pp. 1129-1136, 1996.

[4] L. A. Lipsitz, I. Nakajima, M. Gagnon, T. Hirayama, C. M. Connelly, I. H., and H. T., "Muscle strength and fall rates among residents of japanese and american nursing homes : an international cross-cultural study," Journal of the American Geriatrics Society, vol. 42, pp. 953-9, 1994.
[5] M. C. Nevitt, S. R. Cummings, S. Kidd, and D. Black, "Risk factors for recurrent nonsyncopal falls. A prospective study," JAMA, vol. 261, no. 18 , pp. 2663-2668, 1989.

[6] M. E. Tinetti, M. Speechley, and S. F. Ginter, "Risk factors for falls among elderly persons living in the community," $N$ Engl $J$ Med, vol. 319 , no. 26 , pp. 1701-1707, 1988.

[7] A. Hayen and R. Mitchell, "A review of injury hospitalisations during 1989-1990 to 2003-2004," NSW Injury Risk Management Research Centre, University of NSW, p. 95, 2006

[8] E. G. project team, "Action on accidents and injuries," Newsletter of the Working Party on Accidents and Injuries, vol. 1, 2005.

[9] B. Connell and S. Wolf, "Environmental and behavioral circumstances associated with falls at home among healthy elderly individuals," Archives of Physical Medicine and Rehabilitation, vol. 78, no. 2, pp. $179-186,1997$

[10] J. M. Hausdorff, H. K. Edelberg, S. L. Mitchell, A. Goldberger, and J. Wei, "Increased gait unsteadiness in community-dwelling elderly fallers," Archives of Physical Medicine and Rehabilitation, vol. 78, no. 3, pp. $278-283,1997$.

[11] E. John, W. Liu, and R. Gregory, "Biomechanics of muscular effort: Age-related changes," Medicine \& Science in Sports \& Exercise, vol. 41, no. 2, pp. 418-425, 2008.

[12] S. Okada, "Investigating a prevention of falling and barrier free : a point of view of maintaining and improving physical fitness and motor function," Bulletin of Graduate School of Human Development and Environment, Kobe University, vol. 1, no. 1, pp. 143-150, 2007.

[13] A. Nardone, J. Tarantola, A. Giordano, and M. Schieppati, "Fatigue effects on body balance," Electroencephalography and Clinical Neurophysiology/Electromyography and Motor Control, vol. 105, no. 4, pp. $309-320,1997$.

[14] K. Yoshino, T. Motoshige, T. Araki, and K. Matsuoka, "Effect of prolonged free-walking fatigue on gait and physiological rhythm," Journal of Biomechanics, vol. 37 , no. 8, pp. 1271 - 1280 , 2004. [Online]. Available: http://www.sciencedirect.com/science/article/ B6T82-4BJWVD5-2/2/56d070f51c274c5e5ece82f5a19aa5a9

[15] S. Garland and A. McComas, "Reflex inhibition of human soleus muscle during fatigue." J Physiol, vol. 429, no. 1, pp. 17-27, 1990.

[16] J. Himann, D. Cunningham, P. Rechnitzer, and D. Paterson, "Age-related changes in speed of walking," Medicine \& Science in Sports \& Exercise, vol. 20, no. 2, pp. 161-6, 1988.

[17] I. Wall, C., M. S. Weinberg, P. B. Schmidt, and D. E. Krebs, "Balance prosthesis based on micromechanical sensors using vibrotactile feedback of tilt," IEEE Trans. on Biomedical Engineering, vol. 48, no. 10, pp. 1153-1161, Oct 2001.

[18] Y. Tsuruoka and R. Shibasaki, "Spectral analysis in walking balance by elderly subjects," Engineering in Medicine and Biology Society, 2006. EMBS '06. 28th Annual International Conference of the IEEE, pp. 5420 5423, Aug. 30 2006-Sept. 32006.

[19] J. Yoo, M. S. Nixon, and C. J. Harris, "Extracting human gait signatures by body segment properties," in Image Analysis and Interpretation, 2002. Proceedings. Fifth IEEE Southwest Symposium on, 2002, pp. 3539.

[20] H. Gu, Q. Ji, and Z. Zhu, "Active facial tracking for fatigue detection," in WACV '02: Proceedings of the Sixth IEEE Workshop on Applications of Computer Vision. Washington, DC, USA: IEEE Computer Society, 2002, p. 137.

[21] K. Shinkoda, "Mechanism of falling," Seminar of preventing falling at Hiroshima, 2002.

[22] Y. Nishijima, T. Kato, H. Nakagawa, M. Yoshizawa, and M. Miyashita, "Postural and electromyographical analysis of gait in the aged person," Walking Research, no. 9, pp. 89-94, 2005.

[23] R. Adler, M. Flanigan, J. Huang, R. Kling, N. Kushalnagar, L. Nachman, C. Wan, and M. Yarvis, "Intel mote 2: an advanced platform for demanding sensor network applications," in SenSys '05: Proceedings of the 3rd international conference on Embedded networked sensor systems. New York, NY, USA: ACM, 2005, pp. 298-298.

[24] gumstix, "Gumstix - dream, design, deliver," April 2009. [Online]. Available: http://www.gumstix.com/

[25] Y. Goto, K. Honma, K. Matsushita, T. Okamoto, and A. Tsujino, "Electromyographic study on grade walking," Annals of physical education, Osaka, vol. 15, pp. 67-76, 1980. 